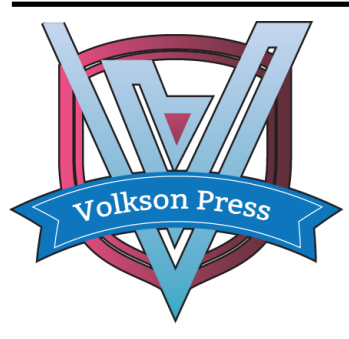

Contents List available at VOLKSON PRESS

New Materials and Intelligent Manufacturing (NMIM)

DOI : http://doi.org/10.26480/icnmim.01.2018.117.120

Journal Homepage: https://topicsonchemeng.org.my/

ISBN: 978-1-948012-12-6

\title{
COMPARISON STUDY ON THE CATALYSIS PERFORMANCE OF MCM-49 AND B ETA ZEOLITE ON THE TRANSALKYLATION OF PIPB
}

\author{
Zhang Yu*, Qin Tizhi, Jiang Di, Wang Duoying, An Duo \\ Jilin Institute of Chemical Technology, Jilin City, China \\ *Corresponding Author Email: zhang99yu@hotmail.com
}

This is an open access article distributed under the Creative Commons Attribution License, which permits unrestricted use, distribution, and reproduction in any medium, provided the original work is properly cited

\section{ARTICLE DETAILS}

Article History:

Received 26 June 2018

Accepted 2 July 2018

Available online 1 August 2018

\section{ABSTRACT}

Zeolite MCM-49 and Beta were synthesized by dynamic hydrothermal method, which were characterized by different techniques, such as XRD, SEM, $\mathrm{N}_{2}$ adsorption-desorption and $\mathrm{NH}_{3}$-TPD. The catalyst performance of MCM- 49 and Beta on the transalkylation of polyisopropyl-benzene (PIPB) had been studied, comparatively. The influence factors of catalytic performance were investigated, such as reaction temperature, raw materials ratio and space velocity. Results indicate that MCM-49 shows better catalytic activity for TIPB under a relatively low reaction temperature, which should be mainly due to a large amount of structurally accessible acid sites caused by its high external surface area.

\section{KEYWORDS}

MCM-49, Beta, Transalkylation, PIPB.

\section{INTRODUCTION}

MCM-49 is a kind of layered microporous materials with MWW topology structure, being widely used in some important industry process, such as alkylation, catalytic cracking, isomerization and disproportionation [110]. Previous studies have shown that the external acid sites play an important role on the catalysis performance of MCM-49 [11-14]. It prohibits a great potential in the catalytic transformation of macromolecules.

Isopropyl-benzene (IPB) is an important organic chemicals, mainly used in the synthesis of phenol and acetone, which is produced by liquid phase alkylation of benzene with propylene. Inevitably, by-products of polyisopropyl-benzene (PIPB) will be obtained including disopropylbenzene (o-DIPB, m-DIPB and p-DIPB) and trisopropylbenzene (TIPB). Traditionally, PIPB could be converted to IPB through transalkylation process.

The catalytic performance of zeolites on the transalkylation of benzene with DIPB had been reported [15]. However, the study on the transalkylation of benzene with PIPB (including both DIPN and TIPB) is rarely. Because of the larger molecules size of TIPB and the diffusion inhibition of zeolite channel, a higher reaction temperature is necessary to obtain a high conversion of TIPB. Unfortunately, according to literature earlier, reaction temperature above $493 \mathrm{~K}$ will bring to obviously increase of n-propylbenzene (NPB), which will decrease the quality of phenol dramatically. So, there is a challenge to explore a kind of catalyst with suitable porous structure, channel size and acid properties, which can conversion both TIPB and DIPB, effectively.

In this work, layered MCM-49 zeolites were prepared by dynamic hydrothermal method. All samples were characterized by means of XRD, SEM, $\mathrm{N}_{2}$-adsorption/desorption and $\mathrm{NH}_{3}$-TPD. The catalytic performance of MCM-49 on the transalkylation of PIPB was investigated compared to Beta.

\section{EXPERIMENTAL}

\subsection{Materials Preparation}

HBeta zeolite $\left(\mathrm{SiO}_{2} / \mathrm{Al}_{2} \mathrm{O}_{3}=20\right)$ was synthesized by dynamic hydrothermal method according to reported previously [16]. NaMCM-49 zeolite was synthesized from a gel having the following composition: $\mathrm{Si} / \mathrm{Al}=10.2$, $\mathrm{Na} / \mathrm{SiO}_{2}=0.15, \mathrm{HMI} / \mathrm{SiO}_{2}=0.30, \mathrm{H}_{2} \mathrm{O} / \mathrm{SiO}_{2}=20$ as reported previously [13]. This gel was crystallized in a Teflon-lined autoclave at $170{ }^{\circ} \mathrm{C}$ for 72 $\mathrm{h}$ with rotating rate of $45 \mathrm{rpm}$. The precursor of MCM- 49 was recovered by filtration, washed with distilled water, dried at ambient temperature and calcined in air at $540{ }^{\circ} \mathrm{C}$ for $6 \mathrm{~h}$ to give the NaMCM- 49 zeolite. The protonic forms of MCM-49 zeolite was obtained by three times exchanges with $1.5 \mathrm{M}$ ammonium acetate solution for $10 \mathrm{~h}$ at $90{ }^{\circ} \mathrm{C}$, followed by calcination in air at $540{ }^{\circ} \mathrm{C}$ for $6 \mathrm{~h}$ to give HMCM- 49 .

\subsection{Catalystic Characterization}

The textural properties of samples were obtained in a Micromeritics ASAP2020 apparatus. X-ray diffraction (XRD) patterns were collected by a Shimadzu XRD-6000 diffractometer using $\mathrm{Cu} K \alpha$ radiation $(\lambda=0.1542 \mathrm{~nm})$. Temperature-programmed desorption of ammonia ( $\mathrm{NH}_{3}-\mathrm{TPD}$ ) was carried out on a Finesorb 3010D chemisorption apparatus [9]. A Zeiss JSM$6490 \mathrm{LV}$ instrument oper-ating a $20 \mathrm{kV}$ was used to perform the scanning electron microscopy (SEM) and the energy dispersive analysis of X-rays (EDAX).

\subsection{Reaction Conditions}

Transalkylation of polyisopropyl-benzene was performed in a fix-bed stainless microreactor. The diameter of the reaction tube is 10 centimeter. The reactor was charged with $1 \mathrm{~g}$ of catalysts crushed and sieved to 40-60 mesh particles. The reaction mixture $\left(\omega_{\mathrm{B}}: \omega_{\mathrm{DIPB}}: \omega_{\text {TIPB }}=50 \sim 100: 25: 7\right)$ was fed to the reactor by a pump. Catalytic tests were carried out under the following conditions: reaction pressure $=3 \mathrm{MPa}$; temperature $=170-230^{\circ} \mathrm{C}$; WHSV $=0.5 \sim 2.5 \mathrm{~h}^{-1}$ 


\section{RESULT AND DISCUSSION}

\subsection{Characterization of Catalysts}

Figure 1 shows the X-ray patterns of as-made MCM-49, calcined MCM-49 and calcined Beta zeolite. All these patterns are in good agreement with those previously reported $[1,16]$. For as-made MCM-49, the diffraction peaks seems sharp, and exits three diffraction peaks at $2 \theta=26^{\circ} \sim 29^{\circ}$ $(\mathrm{d}=0.343 \sim 0.308 \mathrm{~nm})$. For calcined MCM-49, it shows an obviously increase in the intensity of diffraction peaks at $15^{\circ}$. It shows that synthesized MCM-49 sample is pure and well crystallized.

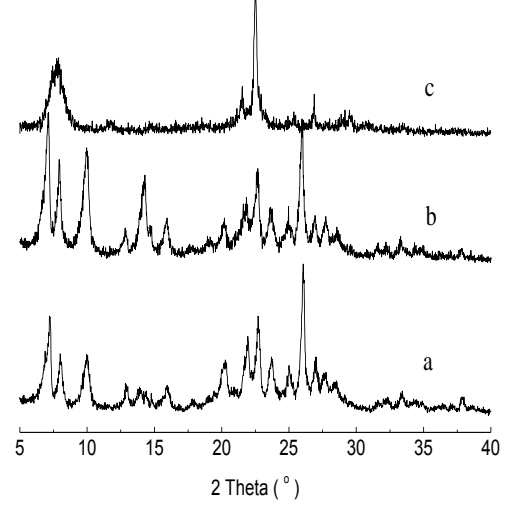

Figure 1: XRD pattern of (a) as-made MCM-49; (b) calcined MCM-49; (c) Beta.
The nitrogen adsorption-desorption isotherms of samples are presented in Figure 2, which indicates that both HMCM-49 and HBeta are microporous materials. The inflection point appears at relative pressure 0.1 . Table 1 gives the textural property of MCM- 49 and Beta zeolite. The BET surface area and external surface area of MCM- 49 is $476 \mathrm{~m}^{2} \cdot \mathrm{g}^{-1}$ and 83 $\mathrm{m}^{2} \cdot \mathrm{g}^{-1}$ respectively. The total pore volume and micropore pore volume is $0.58 \mathrm{~cm}^{3} / \mathrm{g}$ and $0.17 \mathrm{~cm}^{3} / \mathrm{g}$. Compared to MCM- 49 , the BET surface area and micropore surface area of Beta is higher, however the external surface area is obviously lower.

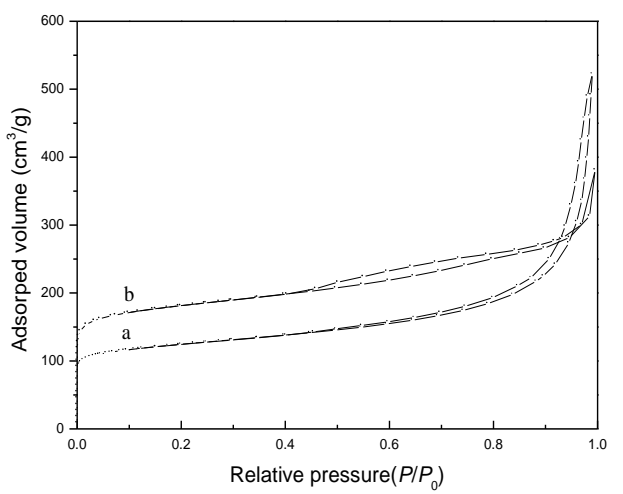

Figure 2: Nitrogen adsorption-desorption isotherm of (a) MCM-49 and (b) Beta.

Table 1: Textural property of MCM-49 and Beta zeolite

\begin{tabular}{|llllll|}
\hline Sample & $\begin{array}{l}\mathrm{S}_{\text {BET }} \\
{\left[\mathrm{m}^{2} / \mathrm{g}\right]}\end{array}$ & $\begin{array}{l}\mathrm{S}_{\text {t-plot micro. }} \\
{\left[\mathrm{m}^{2} / \mathrm{g}\right]}\end{array}$ & $\begin{array}{l}\mathrm{S}_{\text {t-plot external }} \\
{\left[\mathrm{m}^{2} / \mathrm{g}\right]}\end{array}$ & $\begin{array}{l}\mathrm{V}_{\text {total }} \\
{\left[\mathrm{cm}^{3} / \mathrm{g}\right]}\end{array}$ & $\begin{array}{r}\mathrm{V}_{\text {t-plot micropore }} \\
{\left[\mathrm{cm}^{3} / \mathrm{g}\right]}\end{array}$ \\
\hline MCM-49 & 476 & 378 & 83 & 0.58 & 0.17 \\
Beta & 580 & 395 & 47 & 0.59 & 0.26 \\
\hline
\end{tabular}

Figure 3 shows the SEM photograph of MCM-49 and Beta. MCM-49 synthesized by dynamic hydrothermal method presents a well dispersed layered morphology. The size of the layered crystal is about 1-2 $\mu \mathrm{m}$ along $a, b$ dimension, and 20-30 $\mathrm{nm}$ along $c$ dimension. For Beta, it shows an irregular particle morphology, and the size of particle agglomerates is about 3-8 $\mu \mathrm{m}$.

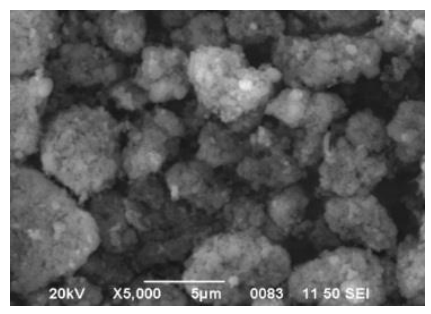

(a)

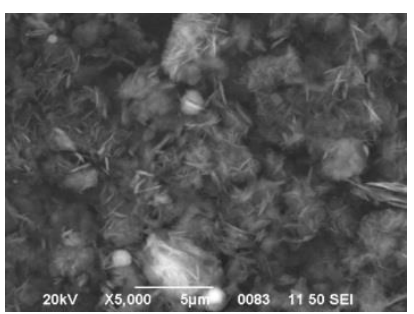

(b)
Figure 3: SEM photograph of (a) Beta and (b)MCM-49

The acid properties of HMCM-49 and HBeta were characterized by temperature-programmed desorption (TPD) of ammonia (see Figure 4). Both $\mathrm{NH}_{3}$-TPD profiles show two peaks at around $225 \stackrel{\circ}{\circ} \mathrm{C}$ and $399{ }^{\circ} \mathrm{C}$, which can be attributed to the presence of weak acidic sites and relatively strong acid sites (mainly Brønsted acidity), respectively. Compared to HBeta, HMCM-49 shows a similar total acid amounts and a bit lower strong acid amounts, which is in accordance with the $\mathrm{Si} / \mathrm{Al}$ mole ratio of two samples.

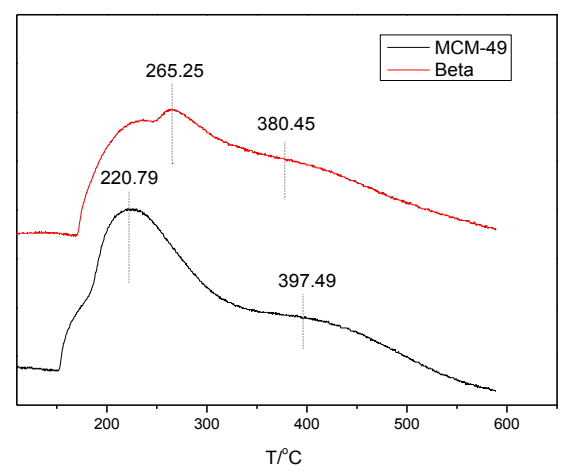

Figure 4: Temperature-programmed desorption of $\mathrm{NH}_{3}$ for (a) $\mathrm{HMCM}-49$ and (b)HBeta

\subsection{Catalytic Performance}

The transalkylation of PIPB with benzene was catalyzed by HMCM-49 and HBeta, respectively. Figure 5 shows the comparison results in the conversion of benzene (CB), DIPB (CD), TIPB (CT), and the yield of IPB (YI) for MCM-49 and Beta. Results indicate that MCM-49 shows a better catalytic activity for the conversion of TIPB, as well as Beta is more suitable for the conversion of DIPB. For Beta zeolite, a higher yield of IPB was obtained.

The effects of reaction temperature on the catalytic performance of MCM49 and Beta zeolite on the transalkylation were investigated in detail (Figure 6). Increase in the reaction temperature would improve the convention of TIPB and the yield of IPB. Results suggest that MCM-49 zeolite shows excellent catalytic activity for the conversion of TIPB under a relatively lower reaction temperature. When the reaction temperature is between 180 to $200{ }^{\circ} \mathrm{C}$, the conversion of TIPB for MCM- 49 is obviously higher than Beta. When the reaction temperature is above $220{ }^{\circ} \mathrm{C}, \mathrm{HMCM}-$ 49 exhibits $100 \%$ convention of TIPB and $72.25 \%$ yield of IPB. 
Figure 7 shows that the mass hour space velocity (MHSV) has a considerable effect on the conversion of TIPB and the yield of IPB. It is observed that the largest yield was obtained when MHSV is $1 \mathrm{~h}^{-1}$. As it is further increased, the conversion and the yield will decrease. The yield of IPB is about $74 \%$, which is similar for both MCM- 49 and Beta.

So, MCM-49 shows a excellent catalytic activity for the conversion of TIPB at a relatively low reaction temperature, which could be attributed to its numerous acid sites on the external surface, which helps to promote the reaction of macromolecules and reduce the diffusion resistance.

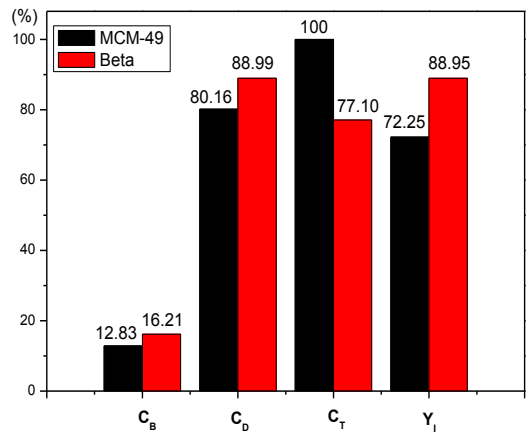

Figure 5: Comparison of the conversion of benzene $\left(C_{B}\right), D I P B\left(C_{D}\right)$, TIPB $\left(\mathrm{C}_{\mathrm{T}}\right)$, and the yield of $\mathrm{IPB}\left(\mathrm{Y}_{\mathrm{I}}\right)$

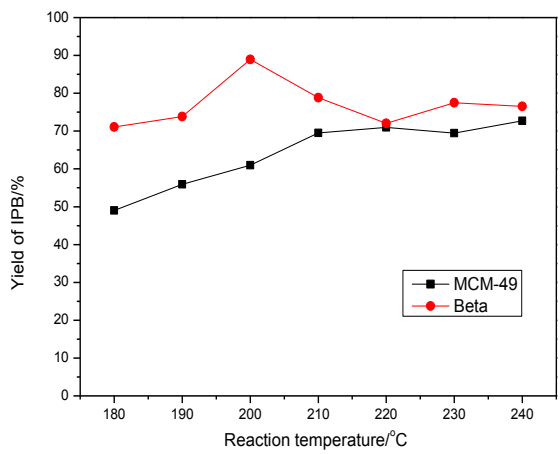

Figure 6: Effect of the reaction temperature on the conversion of TIPB and the yield of IPB $\left(P=1.8 \mathrm{MPa}, \omega_{\mathrm{B}}: \omega_{\mathrm{PIPB}}=2.3, \mathrm{WHSV}=1 \mathrm{~h}^{-1} \mathrm{~T} .0 . \mathrm{S} .=10 \mathrm{~h}\right)$
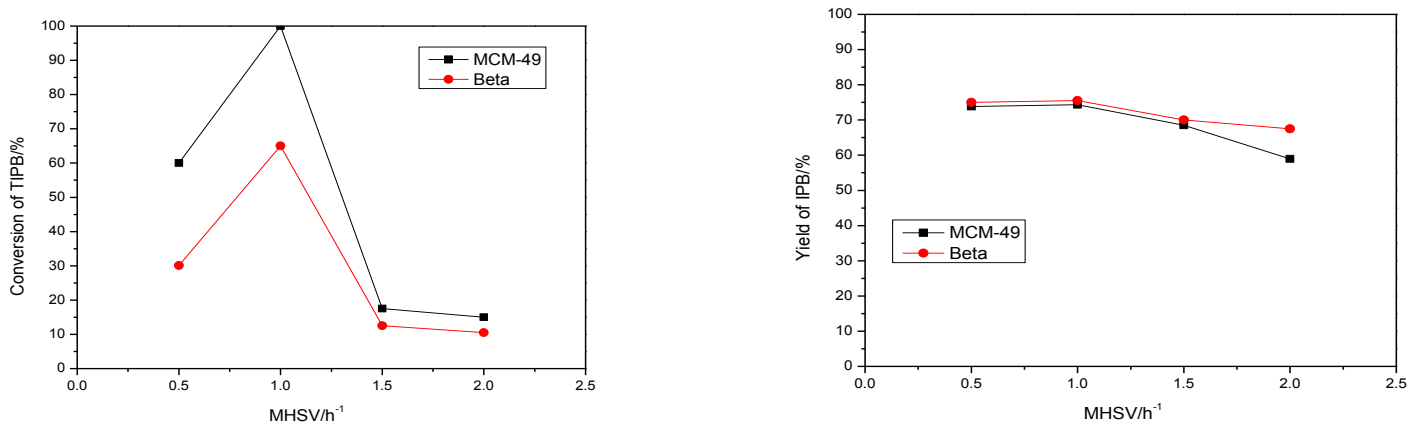

Figure 7: Effect of the mass space velocity on the conversion of TIPB and the yield of IPB $\left(\mathrm{P}=1.8 \mathrm{MPa}, \mathrm{T}=220^{\circ} \mathrm{C}, \omega_{\mathrm{B}}: \omega_{\mathrm{PIPB}}=2.3\right.$, T.0.S. $\left.=10 \mathrm{~h}\right)$

\section{CONCLUSIONS}

Pure and well crystallized MCM-49 and Beta zeolite have been synthesized by dynamic hydrothermal method. XRD and SEM reveals that MCM-49 presents a well dispersed layered morphology. The characterization of textural and acid properties shows a exiting of higher external surface area for MCM-49 zeolite and a similar acid strength. MCM- 49 shows a better catalytic activity for the conversion of TIPB at a relatively low reaction temperature, and Beta is more suitable for the conversion of DIPB. The catalytic performance of MCM-49 could be attributed to the exiting of numerous acid sites on the external surface, which promote the reaction of macromolecules and reduce the diffusion resistance.

\section{ACKNOWLEDGEMENT}

We are grateful to Technological Innovation Development Project of Jilin city (20161205) and Technological Development Project of Jilin Institute of Chemical Technology (2015018) for financial support.

\section{REFERENCES}

[1] Bennett, J.M., Chang, C.D., Lawton, S. L.1993. Synthetic porous crystalline MCM-49, its synthesis and use, US 5236575.

[2] Lawton, S.L., Fung, A.S., Kennedy. 1996. Zeolite MCM-49: A ThreeDimensional MCM-22 Analogue Synthesized by in Situ Crystallization. The Journal of Physical Chemistry A, 100 (9), 3788-3798
[3] Karsten, E., Rudolf, K. 1998. Preparation of amines from olefins on zeolites of the MCM-49 or MCM-56 type, US 5840988.

[4] Cheng, J.C., Huang, T.J. 1996. Process for the alkylation of benzene-rich reformate using MCM-49. US 5545788.

[5] Stephen, H.B., Robert, A.C., Lorenzo De, C. 1999. Ethyl acetate synthesis from ethylene and acetic acid using solid acid catalysts, US 5973193.

[6] Husain, A., Albin, H.J., Donald, K.J. 1993. Isoparaffin: olefin alkylation in the presence of synthetic porous MCM-49, US 5254792.

[7] Gao, N.N., Xie, S.J., Liu, S.L. 2014. Catalytic Degradation of LDPE and PP over MCM-49 Based Micro-Mesoporous Composites. Catalysis Letters, 144, 1296-1304.

[8] Xing, E.H., Shi, Y.C., Zheng, A.G. 2015. Transformation from NaA to MCM-49 Zeolite and Its Catalytic Alkylation Performance. Industrial \& Engineering Chemistry Research, 54 (12), 3123-3135.

[9] Shi, Y.C., Xing, E.H., Xie, W.H. 2016. Shape selectivity of Beta and MCM49 zeolites in liquid-phase alkylation of benzene with ethylene. Journal of Molecular Catalysis a-Chemical. 418, 86-94.

[10] Yao, S.D., Sun, C.Y., Li, J. 2009. Reaction Coupling of Methane Steam Reforming and Methane Dehydroaromatization for Improving Durability of Mo/MCM-49 Catalyst. Chinese Journal of Catalysis, 30 (10), 1022-1028. 
[11] Jin, M., Shang, Y.C., Yang, P.P., Jia, M.J., Zhang, W.X., Wu, T.H. 2008. Acid properties and catalytic performance for phosphonic acid modified MCM49 zeolites in skeletal isomerization of n-butene. Catalysis Communications, 10 (2),146-149.

[12] Wei, L.G., Shang, Y.C., Yang, P.P. 2008. Alkylkylation of phenol with isopropanol over MCM-49 zeolites. Reaction Kinetics and Catalysis Letters, 93 (2), 265-271.

[13] Zhang, Y., Wang, L.X., Wu, S.J. 2007.Structure and acid properties of phosphate-modified MCM-49 zeolites and their catalysis performance for the alkylation of benzene with propylene in liquid. Chemical Journal of Chinese Universities-Chinese, 28 (9), 1726-1730.
[14] Yang, P.P., Wang, Z.L., Yu, J.F. 2005. Preparation, characterization of MCM-49 and catalytic performance of Pd/MCM-49 catalysts in one-step synthesis of MIBK from acetone. Polish Journal of Chemistry, 79 (1), 121127.

[15] Pradhan, A.R., Rao, B.S. 1993. Transalkylation of di-isopropylbenzene over large pore zeolites [J]. Applied Catalysis A: General, 106, 143-153.

[16] He, M.Y., Chen, Q., Yang, X.J., Lu, L.D., Wang, X. 2003. Synthesis of nonnylphenol from dinonanylphenol and phenol calalyze by thermostable cation exchange resin. Ion Exchange and Adsorption, 5, 444-449

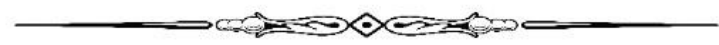

